\title{
Pancreatic Neuroendocrine Tumor Producing Insulin and Vasopressin
}

Omalkhaire M. Alshaikh, Ju-Yoon Yoon, Bryan A. Chan, Monika K. Krzyzanowska, Jagdish Butany, Sylvia L. Asa, Shereen Ezzat

\begin{abstract}
The objective of the study is to report a rare case of pancreatic neuroendocrine tumor (pNET) producing insulin and vasopressin. We describe the clinical presentation and management of a metastatic PNET with refractory hypoglycemia and progressive severe hyponatremia. A 52-year-old patient had abdominal pain leading to the diagnosis of a tumor that was initially presumed to be splenic in origin. Investigations ultimately identified a pancreatic mass that on biopsy proved to be a PNET. Eventually, he developed extensive liver metastases, and with tumor progression, he manifested hypoglycemia and severe hyponatremia. He was managed with multiple therapies including somatostatin analogue, peptide-receptor-radionuclide-therapy (PRRT), diazoxide, and everolimus; none of these therapeutic modalities was successful in controlling functional and structural progression of the tumor. Ultimately, the PNET proved fatal and autopsy confirmed widely metastatic disease that stained strongly and diffusely for vasopressin, a feature not seen in the previous liver biopsy. This case illustrates the challenges of diagnosis and management of aggressive insulin-producing pNETs and highlights the potential concomitant ectopic production of vasopressin leading to refractory
\end{abstract}

\section{Introduction}

Insulin-producing pancreatic neuroendocrine tumors (pNETs) are relatively rare, with an estimated incidence of approximately 1-3 per million per year. Only $10 \%$ of these tumors are thought to follow a malignant course [1]. The paraneoplastic syndrome secondary to ectopic vasopressin production by tumor cells, known as the syndrome of inappropriate ADH (SIADH), is most commonly associated with lung tumors, usually small-cell carcinomas that are aggressive poorly differentiated neuroendocrine malignancies [2]. Under the broad umbrella of neuroendocrine tumors (NETs), olfactory neuroblastomas and gastrointestinal NETs have occasionally been reported to give rise to SIADH $[3,4]$. In rare cases, embolization of a NET has been associated with ectopic vasopressin leading to SIADH [5]. To our knowledge, combined insulin and ectopic vasopressin production by a PNET has not been previously reported. 
We report an unusual case of a malignant PNET which, with disease progression, acquired the ability to secrete insulin in excess and to ectopically produce vasopressin resulting in the SIDAH.

\section{Case Presentation}

A 52-year-old man presented with intermittent abdominal pain. He maintained normal appetite, weight, and physical activity. Family history was negative for endocrine neoplasia. Abdominal and systemic examinations were normal. Biochemistry reveled hyperbilirubinemia, iron deficiency anemia, and thrombocytopenia. Other blood tests including fasting blood sugar, serum electrolytes, and chromogranin A were normal. Because of the anemia, upper and lower gastrointestinal endoscopies were performed but revealed no pathologies. However, unresolved abdominal pain prompted abdominal ultrasound and computed tomographic (CT) scanning of the abdomen that demonstrated a suspicious mass in the splenic hilum associated with splenomegaly and thrombosis of the portal, superior mesenteric and splenic veins. Anticoagulant therapy was commenced. Surgical resection was deemed not feasible.

On follow-up 1 year later, serum chromogranin A had increased to $161 \mu \mathrm{g} / \mathrm{L}$ (normal <50 $\mu \mathrm{g} / \mathrm{L}$ ); glucose was normal at $6.5 \mathrm{mmol} / \mathrm{L}$ and he had a normal 24-h urinary $5 \mathrm{HIAA}$ level of $26 \mu \mathrm{mol} /$ day (Fig. 1a; upper). Initial serum sodium level was $140 \mathrm{mmol} / \mathrm{I}$. A few months later, he had mild hyponatremia (serum sodium of $132 \mathrm{mmol} / \mathrm{l}$, normal 135-145). Endocrine causes of hyponatremia including adrenal insufficiency and hypothyroidism were ruled out. Hyponatremia corrected without specific intervention (Fig. 1a; middle). Abdominal CT imaging showed increase in size of the pancreatic/splenic mass to $7.6 \mathrm{~cm}$ (Fig. 1a; lower). This mass was avid on FDG PET scanning. Core biopsy confirmed a NET (Fig. 2a, b) with positivity for chromogranin A (Fig. 2c) and synaptophysin; it was consistent with pancreatic origin, as assessed by expression of low molecular weight keratin (LMWK), CK19 and CK20, while being negative for CK7, TTF-1, and CDX2. It was a Grade 1 lesion, with a mitotic count of two per ten high-power fields (HPFs) and Ki-67 index of approximately $1 \%$. Importantly, this pancreatic NET (pNET) showed diffuse expression of pancreatic polypeptide (Fig. 2d) and focal staining for insulin (Fig. 2e); it was negative for glucagon, somatostatin, PYY, and VIP. All of these immunohistochemical tests performed in the clinical laboratory had appropriate positive and negative controls. Octreotide scanning showed avid disease in the pancreas and spleen. The disease was again deemed to be non-operable due to major blood vessel invasion. 
A
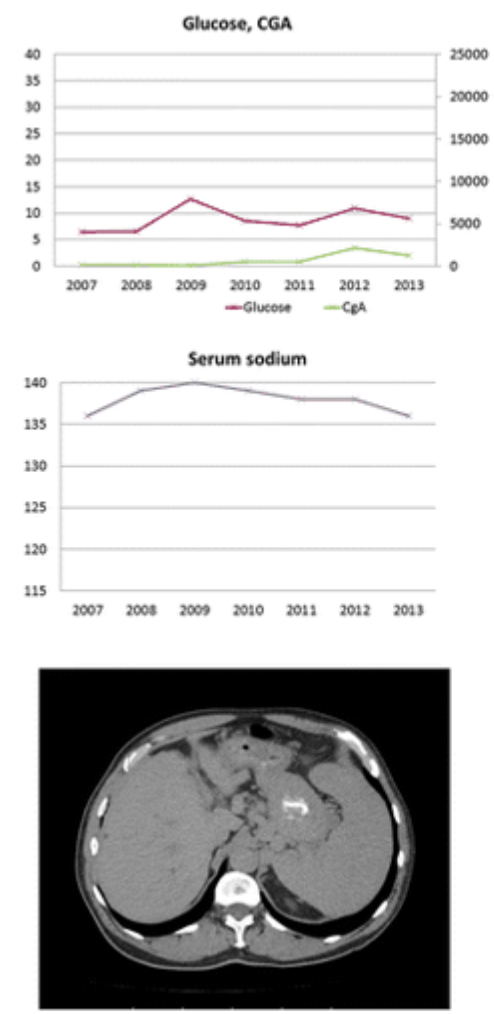

B
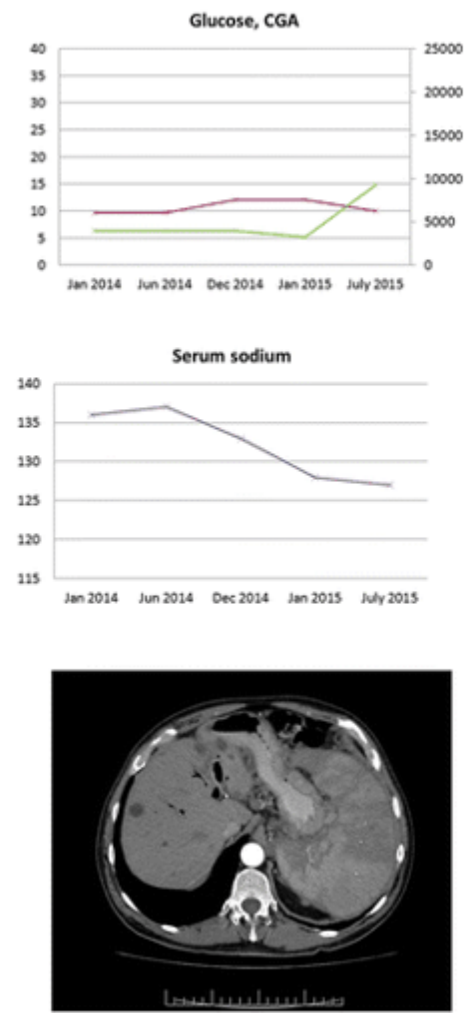

$\mathrm{C}$
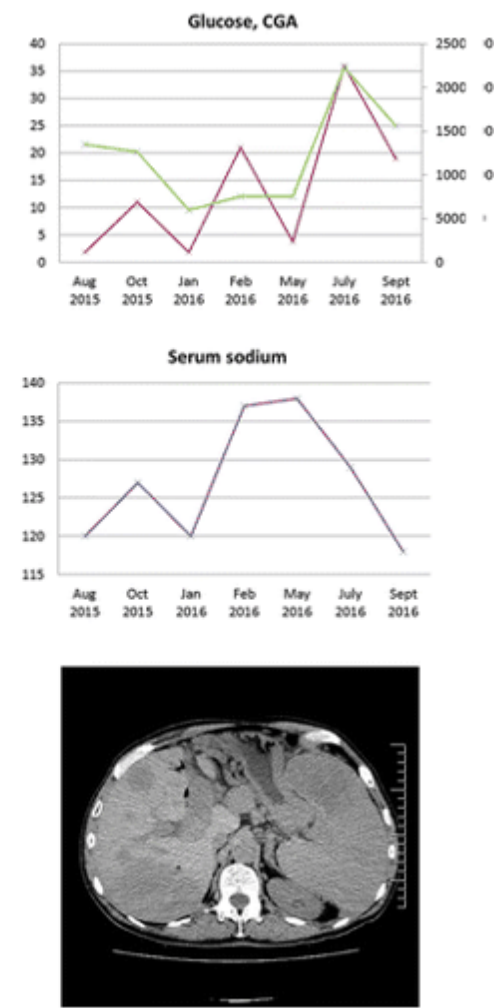

Fig. 1: Biochemical and radiological progression of pNET. a Early stage disease. Patient had normal glucose, mild elevation of serum chromogranin A ( $\mathrm{CgA}$ ) and mild hyponatremia of $132 \mathrm{mmol} / \mathrm{l}$. Computed tomography (CT) imaging shows a 7.6$\mathrm{cm}$ exophytic pancreatic
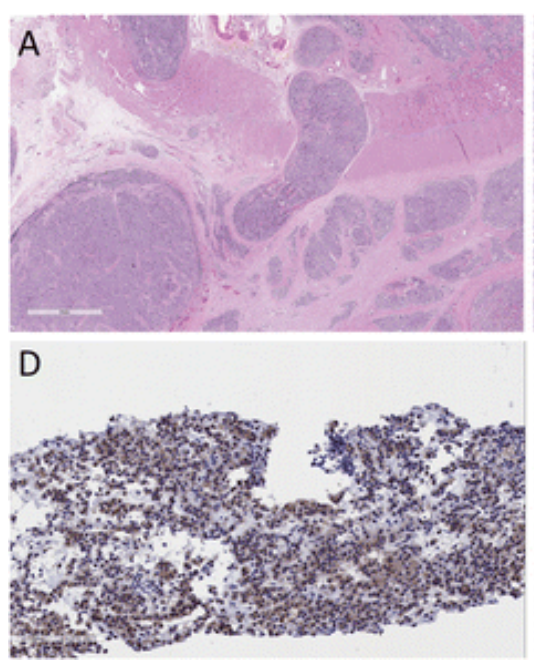
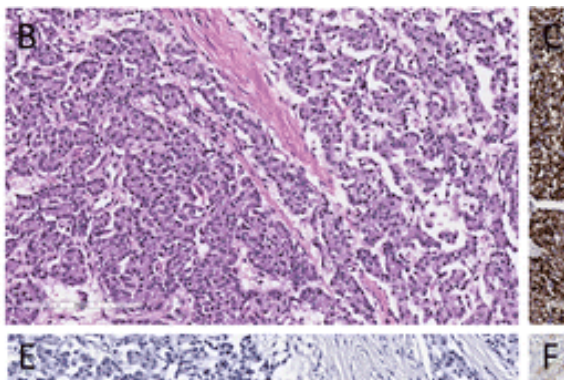
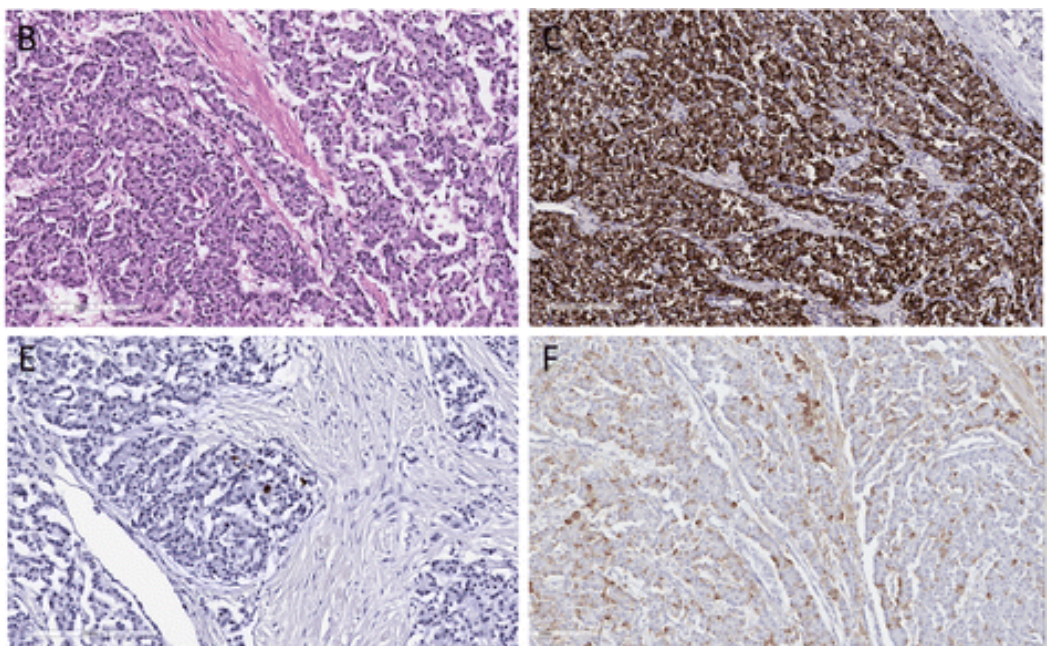

Fig. 2: Pathology of pancreatic neuroendocrine tumor (pNET). The pancreatic neuroendocrine tumor was a widely invasive lesion (a) composed of well-differentiated neuroendocrine cells forming small nests and cords of relatively monotonous cells with uniform "salt

One year later, disease progression with liver metastasis necessitated pancreatic duct stenting and the initiation of octreotide-LAR therapy (Fig. 1b; lower). Two years later, continued disease progression led to the administration of four cycles of peptide radioreceptor therapy (PRRT) that resulted in modest shrinkage of the pancreatic, splenic, and liver tumor deposits. Of note, he 
developed diabetes mellitus after the first cycle of PRRT requiring treatment with the oral hypoglycemic, gliclazide.

Twenty months following PRRT completion, he started to experience hypoglycemia for the first time. Exogenous antidiabetic medications were discontinued. Nevertheless, he continued to experience hypoglycemia with documented blood glucose of $1.8 \mathrm{mmol} / \mathrm{I}$ (normal 3.8-6.0) accompanied by inappropriately elevated pro-insulin of 290 pmol/I (normal <18.8), insulin of 249 pmol/I (normal 13143), and C-peptide of $1195 \mathrm{pmol} / \mathrm{I}$ (normal 370-1470). Serum chromogranin A level increased to $3900 \mu \mathrm{g} / \mathrm{L}$. These hypoglycemic episodes were refractory to intravenous dextrose and dexamethasone. He required prolonged and frequent hospital admissions. Combination therapy with diazoxide at an average dose of $600 \mathrm{mg} /$ day and the mTOR inhibitor everolimus of $10 \mathrm{mg} /$ day were effective in maintaining blood sugars to a level of $4 \mathrm{mmol} / \mathrm{l}$ (Fig. 1b; upper). However, 4 weeks after initiation of this combination therapy, he developed generalized edema and hyponatremia with serum sodium of $129 \mathrm{mmol} / \mathrm{l}$ (135-145 mmol/l). While serum osmolality was $250 \mathrm{mOsm} / \mathrm{kg}$, urine osmolality was $140 \mathrm{mOsm} / \mathrm{kg}$ consistent with inappropriate antidiuretic hormone (SIADH) (Fig. $1 \mathrm{~b}$; middle). Unfortunately, this hyponatremia was not responsive to fluid restriction and furosemide. Diazoxide was considered as a potential cause of this worsening hyponatremia, so the daily dose was reduced to $100 \mathrm{mg}$. Nevertheless, hypoglycemic control worsened with intermittent episodes of blood glucose levels of $3.5 \mathrm{mmol} / \mathrm{l}$. Serum chromogranin A level increased to 15,600 $\mu \mathrm{g} / \mathrm{L}$ (Fig. 1C; upper). Diazoxide was held, but in spite of gradual resolution of volume overload and normalization of blood glucose, the hyponatremia worsened reaching $119 \mathrm{mmol} / \mathrm{l}$ (Fig. 1c; middle). His mental status and overall performance status deteriorated. Structural imaging revealed continued rapid disease progression (Fig. 1c; lower). The patient and his family chose to discontinue aggressive management and he succumbed to the disease nearly 9 years after initial diagnosis.

Autopsy performed 7-h post mortem confirmed a disseminated NET involving the pancreas, liver, spleen, and retroperitoneal tissues. As with the previous biopsy, and using similar protocols in the clinical laboratory, the NET showed expression of chromogranin, synaptophysin, LMWK and CK19, and focal insulin, consistent with the previous biopsy (Fig. 2e); however, unlike the previous biopsy, it was negative for PP, indicating loss of this differentiated function. It was also negative for glucagon, somatostatin, PYY, gastrin, serotonin, CCK, VIP, and TTF-1. In contrast to the previous lesion, however, the recurrent disease was much more proliferative, as judged by mitotic count of five per $10 \mathrm{HPFs}$ (using phospho-histone $\mathrm{H} 3$ staining) and Ki-67 index of 3.5\%, therefore, was 
classified as Grade 2. Given the clinical history of SIADH, staining for vasopressin was performed on both the autopsy sample and the initial tissue biopsy using the same protocol (antibody T-4563, Peninsula 1:300 for $1 \mathrm{~h}$ after citrate pretreatment). The autopsy lesion was diffusely positive for vasopressin (Fig. 2f), whereas the initial tissue biopsy was negative for vasopressin.

\section{Discussion}

We report an unusual case of a pNET with multiple atypical features of interest.

Our patient presented with a splenic mass. This is known to be a confounding feature of pNETs and underscores the importance of continued investigation in establishing the correct diagnosis of a patient with an abdominal mass [6].

Our patient had a tumor producing insulin that was initially clinically non-functioning but ultimately produced clinical features of insulin excess. Endogenous hyperinsulinism usually presents as Whipple's triad. Other uncommon insulinoma presentations include post prandial hypoglycemia, behavioral abnormalities, and refractory seizures [7]. Almost all insulinoma patients present with fasting hypoglycemia, with inappropriately elevated pro-insulin, C-peptide and insulin [1]. However, a normal insulin and C-peptide level does not exclude the diagnosis of an insulinoma, since there are tumors that secrete proinsulin and have normal insulin levels due to the limitations of new, highly specific insulin assays that do not detect proinsulin $[8,9,10]$. Our patient had an unusual initial presentation with no hormone excess and normal biochemical markers, consistent with a clinically non-functioning PNET. In a very unusual clinical manifestation, the tumor, which was focally immunoreactive for insulin on pathology, spontaneously produced clinical features of insulinoma with disease progression years after presentation [11].

Gene expression of insulin is now a well-understood phenomenon that relies on both pancreatic islet-restricted (including PDX-1) and ubiquitous (including CREB) transcription factors [12]. In insulin-producing pNETs, the physiologic regulation of insulin gene expression becomes dysregulated. The loss of menin (gene product of the MEN1 gene), promotes insulin gene promoter activity, as well as contributing to tumorigenesis $[13,14]$. In sporadic cases, recurrent somatic T372R mutations in the transcription factor YY1 have been found in 30\% of insulin-producing pNETs [15], 
but the exact mechanism as to how the YY1 T372R mutation contributes to insulin production remains unclear. Only $10 \%$ of insulinomas have metastatic behavior, usually associated with primary tumor size larger than $3 \mathrm{~cm}$.

Surgical management is the gold standard therapy for functional insulinomas, which tend to present when small and surgically resectable. However, resection may not be an option for large pNETs, especially with vascular invasion [1]. When a lesion cannot be resected, blood glucose management of diabetic patients harboring an underlying insulin-producing PNET can be challenging, since neoplastic secretion of insulin and its precursors may be variable. Our patient was found to have elevated levels of proinsulin, insulin and C-peptide during episodes of hypoglycemia. This was documented well after he had been off his anti-diabetic medications. Diazoxide has been the traditional agent for managing symptomatic hypoglycemia in patients with inoperable insulinoma. More recently, the mTOR inhibitor everolimus has been approved for treatment of pNETs regardless of their hormone functionality. Zhan et al. reported elevated p-mTOR levels in insulinoma compared to normal pancreatic tissue and reported significant reduction in insulin levels and increase in apoptosis in all in vitro insulinomas treated with the mTOR inhibitor rapamycin or the dual $\mathrm{PI3K} / \mathrm{mTOR}$ inhibitor NVP-BEZ235 [16].

Hyponatremia associated with SIADH is most often associated with small-cell lung cancer (SCLC). Other tumors that have been associated with ectopic vasopressin production include ovarian, lung, breast, and prostate cancer $[17,18]$. Arginine vasopressin (AVP) receptors have been reported to be expressed in other endocrine tumors such as pituitary and adrenal cortisol-producing tumors [19]. The mechanism of ectopic vasopressin expression in PNETs remains largely unknown. The interesting observation from our case is the transition in hormone expression during the course of PNET tumor progression. We hypothesize at least two mechanisms underlying this phenomenon. While a number of common transcription factors play a role in modulating the expression of both insulin and vasopressin, notably CREB, epigenetic modulation is another important shared mechanism for their expression, and a number of microRNAs have been implicated in modulating both insulin and vasopressin expression [20]. In mice, pancreatic acinar cells can be directly converted to insulinproducing beta-cells by combined actions of three transcription factors, namely PDX-1, along with Neurog3 and MafA. In a similar manner, pNET cells may undergo transcriptional reprogramming that result in altered expression profiles of transcription factors. Pancreatic NETs are also epigenetically dysregulated tumors, with the most frequently mutated genes in PNETs being those that function in 
chromatin remodeling, including MEN1, DAXX, and ATRX [21]. Further dysregulation in the epigenome of NETs may have resulted in and/or contributed to both tumor progression and altered hormone switching during the transition.

In other solid tumors, hyponatremia is a negative prognostic feature associated with shorter survival duration [2]. However, there is a misconception that AVP is the main underlying cause of hyponatremia in this setting. AVP itself may not be sufficient to cause hyponatremia in the absence of increased total body water and a defect in the ability to dilute urine and excrete free water. The osmoregulatory defect in malignancy is characterized by significant elevations in plasma AVP that fluctuate widely, irrespective of the increases in plasma osmolality or sodium produced by hypertonic saline infusion [22]. Nevertheless, it is clear that some malignancies can secrete AVP in excess. Key et al. described elevated plasma AVP concentrations in patients with pheochromocytoma [22]. Periodic production of ectopic AVP by a NET may explain the substantial and slow decline of serum sodium levels as in our patient.

Neurological symptoms including cerebral edema may be the earliest sign of acute hyponatremia. Adaptive mechanisms of the brain to limit cerebral edema by extruding sodium, potassium salts and then organic solutes as glutamate, myoinositol could explain the asymptomatic compensation for chronic hyponatremia [23]. Fluid restriction as an initial treatment may be effective to correct hyponatremia, however rigorous interventions such as hypertonic saline are eventually required. Clinical use of Vasopressin receptor 2 antagonists have been evaluated in this clinical setting. Tolvaptan and Conivaptan have been approved by the European Medicines Agency and FDA for hyponatremia treatment in hypervolemic or euvolemic hyponatremia. Tolvaptan has been evaluated in the Ascending Levels of Tolvaptan in Hyponatremia (SALT) trial at doses of 15-60 mg/day for 4-30 days. Effectiveness was demonstrated by increases in serum sodium level within $24 \mathrm{~h}$ after administration with limited major side effects. Importantly, there was no need for fluid restriction [24]. Intriguingly, AVP receptor agonists have been reported to diminish neuroendocrine tumor expression and growth of human lung and prostate tumor cell lines [18]. Taken together, these findings raise the question of whether ectopic AVP expression may reflect a compensatory response to NET tumor progression. 
In summary, our case is unique in multiple ways: our patient presented with a PNET that mimicked a splenic lesion. He initially had a clinically nonfunctioning tumor that with tumor progression, started to produce insulin years after his initial presentation. He was diabetic, creating additional complexities in the management of an unresectable source of insulin-excess. Ectopic AVP production with hyponatremia coincided with tumor progression. Systematic prospective examination of SIADH in NET disease progression should clarify the true frequency of this intriguing phenomenon.

\section{References}

1. de Herder WW, Niederle B, Scoazec JY, Pauwels S, Kloppel G, Falconi M, et al. Well-differentiated pancreatic tumor/carcinoma: insulinoma. Neuroendocrinology. 2006;84(3):183-188.

2. Castillo JJ, Vincent M, Justice E. Diagnosis and management of hyponatremia in cancer patients. Oncologist. 2012;17(6):756-765.

3. Kunc M, Gabrych A, Czapiewski P, Sworczak K. Paraneoplastic syndromes in olfactory neuroblastoma. Contemp Oncol (Pozn). 2015;19(1):6-16.

4. Hwang K, Jeon D-H, Jang HN, Bae EJ, Lee JS, Cho HS, et al. Inappropriate antidiuretic hormone syndrome presenting as ectopic antidiuretic hormone-secreting gastric adenocarcinoma: a case report. Journal of Medical Case Reports. 2014;8:185.

5. Yarmohammadi H, Erinjeri JP, Brown KT. Embolization of metastatic neuroendocrine tumor resulting in clinical manifestations of syndrome of inappropriate secretion of antidiuretic hormone. J Vasc Interv Radiol. 2015;26(4):533-537.

6. Ezzat S. PANCREATIC INCIDENTALOMAS: IS IT NET OR NOT? Endocrine practice : official journal of the American College of Endocrinology and the American Association of Clinical Endocrinologists. 2016;22(7):895-896.

7. Shreenivas AV, Leung V. A Rare Case of Insulinoma Presenting with Postprandial Hypoglycemia. The American Journal of Case Reports. 2014;15:488-491.

8. Fadini GP, Maran A, Valerio A, Meduri F, Pelizzo M, Miotto D, et al. Hypoglycemic Syndrome in a Patient with Proinsulin-Only Secreting Pancreatic Adenoma (Proinsulinoma). Case Reports in Medicine. 2011;2011:1-5.

9. Gomez-Perez FJ, Cuevas-Ramos D, Valdes PA, Aguilar-Salinas CA, Mehta R, Rull JA. Beta-cell adenomas without hyperinsulinemia with use of highly specific insulin radioimmunoassays: case 
report and review of literature. Endocrine practice : official journal of the American College of Endocrinology and the American Association of Clinical Endocrinologists. 2010;16(4):660-663.

10. Carneiro DM, Levi JU, Irvin GL, 3rd Rapid insulin assay for intraoperative confirmation of complete resection of insulinomas. Surgery. 2002;132(6):937-942; discussion 42-3.

11. Kamocki ZK, Wodynska NA, Pryczynicz A. Co-existence of insulinoma and diabetes: A case report. Oncol Lett 2014;8(4):1697-1700.

12. Melloul D, Marshak S, Cerasi E. Regulation of insulin gene transcription. Diabetologia 2002;45(3):309-326.

13. Biondi CA, Gartside MG, Waring P, Loffler KA, Stark MS, Magnuson MA, et al. Conditional Inactivation of the Men1 Gene Leads to Pancreatic and Pituitary Tumorigenesis but Does Not Affect Normal Development of These Tissues. Molecular and Cellular Biology. 2004;24(8):3125-3131.

14. Balogh K, Rácz K, Patócs A, Hunyady L. Menin and its interacting proteins: elucidation of menin function. Trends in Endocrinology \& Metabolism. 2006;17(9):357-364.

15. Cao Y, Gao Z, Li L, Jiang X, Shan A, Cai J, et al. Whole exome sequencing of insulinoma reveals recurrent T372R mutations in YY1. Nat Commun. 2013;4:2810.

16. Zhan HX, Cong L, Zhao YP, Zhang TP, Chen G, Zhou L, et al. Activated mTOR/P70S6K signaling pathway is involved in insulinoma tumorigenesis. J Surg Oncol. 2012;106(8):972-980.

17. Taskin M, Barker B, Calanog A, Jormark S. Syndrome of inappropriate antidiuresis in ovarian serous carcinoma with neuroendocrine differentiation. Gynecologic Oncology. 1996;62(3):400-404.

18. Pifano M, Garona J, Capobianco CS, Gonzalez N, Alonso DF, Ripoll GV. Peptide Agonists of Vasopressin V2 Receptor Reduce Expression of Neuroendocrine Markers and Tumor Growth in Human Lung and Prostate Tumor Cells. Front Oncol. 2017;7:11.

19. Louiset E, Contesse V, Groussin L, Cartier D, Duparc C, Perraudin V, et al. Expression of vasopressin receptors in $\mathrm{ACTH}$-independent macronodular bilateral adrenal hyperplasia causing Cushing's syndrome: molecular, immunohistochemical and pharmacological correlates. J Endocrinol. 2008;196(1):1-9.

20. Eliasson L, Esguerra J. Role of non-coding RNAs in pancreatic beta-cell development and physiology. Acta Physiologica. 2014;211(2):273-284. 
21. Jiao Y, Shi C, Edil BH, de Wilde RF, Klimstra DS, Maitra A, et al. DAXX/ATRX/MEN1, and mTOR Pathway Genes Are Frequently Altered in Pancreatic Neuroendocrine Tumors. Science. 2011;331(6021):1199-1203.

22. Robertson GL. Regulation of arginine vasopressin in the syndrome of inappropriate antidiuresis. Am J Med. 2006;119(7 Suppl 1):S36-S42.

23. Sterns RH, Silver SM. Brain volume regulation in response to hypo-osmolality and its correction. Am J Med. 2006;119(7 Suppl 1):S12-S16.

24. Cardenas A, Gines P, Marotta P, Czerwiec F, Oyuang J, Guevara M, et al. Tolvaptan, an oral vasopressin antagonist, in the treatment of hyponatremia in cirrhosis. Journal of hepatology. 2012;56(3):571-578. 